\title{
Primary squamous cell carcinoma of the salivary gland: immunohistochemical analysis and comparison with metastatic squamous cell carcinoma
}

\author{
Uiree $\mathrm{Jo}^{1}$, Joon Seon Song ${ }^{1}$, Seung-Ho Choi ${ }^{2}$, Soon Yuhl Nam², Sang Yoon $\mathrm{Kim}^{2}$, Kyung-Ja Cho ${ }^{1}$ \\ Departments of ${ }^{1}$ Pathology and ${ }^{2}$ Otorhinolaryngology-Head and Neck Surgery, Asan Medical Center, University of Ulsan College Medicine, Seoul, Korea
}

\begin{abstract}
Background: Primary squamous cell carcinoma (SCC) of the salivary gland is a rare disease, and distinguishing primary SCC from metastatic SCC is difficult. This study investigated the histological and immunohistochemical differences between primary and metastatic salivary gland SCC to improve the accuracy of diagnosis and to explore the pathogenesis of this disease. Methods: Data of 16 patients who underwent surgery for SCC of salivary glands between 2000 and 2018 at Asan Medical Center were retrieved. Eight patients had a history of SCC at other sites, and eight patients had only salivary gland SCC. Immunostaining for p16, p53, androgen receptor (AR), gross cystic disease fluid protein 15 (GCDFP-15), and c-erbB2, as well as mucicarmine staining, were compared between the two groups. Results: Most tumors were located in the center of the salivary glands with extraparenchymal extension. The histology of primary SCC of the salivary gland was consistent with moderately differentiated SCC with extensive desmoplastic reaction and peritumoral inflammation. Involvement of the salivary gland ducts and transition into the ductal epithelium were observed in two cases. Metastatic SCC resembled the primary tumor histologically and was associated with central necrosis. Both groups exhibited negative mucin staining. Two, one, and one primary SCC case exhibited AR, GCDFP-15, and c-erbB2 positivity, respectively. Conclusions: A subset of primary SCCs originated in salivary ducts or was related to salivary duct carcinoma. Distinguishing primary from metastatic SCC of the salivary gland is difficult using histologic features and immunoprofiles. A comprehensive review of the medical history is essential.
\end{abstract}

Key Words: Primary squamous cell carcinoma; Metastatic squamous cell carcinoma; Salivary gland

Received: May 19, 2020 Revised: July 16, 2020 Accepted: July 17, 2020

Corresponding Author: Kyung-Ja Cho, MD, PhD, Departments of Pathology, Asan Medical Center, University of Ulsan College of Medicine, 88 Olympic-ro 43-gil, Songpa-gu, Seoul 05505, Korea

Tel: +82-2-3010-4545, Fax: +82-2-472-7898, E-mail: kjc@amc.seoul.kr

Primary squamous cell carcinoma (SCC) of the salivary gland is a rare disease, accounting for $1.6 \%(0.3 \%-6.9 \%)$ of salivary gland neoplasms [1-5]. The salivary gland is histologically composed of secretory, ductal, and myoepithelial cells. Various neoplasms exhibit secretory, ductal, or myoepithelial differentiation. Therefore, in patients with SCC in the salivary gland, the possibility of metastasis or direct invasion from the adjacent skin or the external auditory canal must be considered $[3,4,6,7]$. In addition, primary SCC must be differentiated from mucoepidermoid carcinoma (MEC) and salivary duct carcinoma using mucicarmine staining and androgen receptor (AR) and gross cystic disease fluid protein 15 (GCDFP-15) immunostainings. Most common events of carcinogenesis are associated with TP53 genetic mutation, and p16 is involving with multiple processes of carcinogenesis in cases of head and neck SCC [8,9]. This study was performed to discover expressional differences of $\mathrm{p} 53$ and p16 immunohistochemical (IHC) stainings between primary SCC and metastatic SCC. Wolff et al. [10] studied c-erbB2 expression in salivary gland carcinoma according to histologic subtype and aggressive behavior. Considering previous results, this study intended to determine the expressional characteristics in primary SCC and metastatic SCC [10-12]. Although the pathogenesis of primary SCC of the salivary gland is unknown, it can be associated with squamous metaplasia of the salivary duct or previous radiation treatment $[4,5]$. The aim of this study was to improve our understanding of the pathology and pathogenesis of primary 
SCC of the salivary gland by comparing its features with those of metastatic SCC.

\section{MATERIALS AND METHODS}

\section{Materials}

Sixteen patients with surgically resected SCC of the salivary gland were identified after searching an anonymized research database at Asan Medical Center between 2000 and 2018. Patient characteristics are listed in Tables 1 and 2. Eight patients (50\%) had primary SCC in the salivary gland. The cases of primary SCC in the salivary gland had no past history of cancer and revealed no other concurrent lesions through imaging studies such as computed tomography (CT) and positron emission tomography. SCC that had been diagnosed at other sites and metastasized to the salivary gland were designated as metastatic SCC in this study. The remaining eight cases (50\%) in this study had a history of SCC at other sites and were classified as metastatic SCC. The clinical parameters collected from the electronic medical records were age at onset, sex, smoking history, survival, and treatment history.

\section{Histologic analysis}

All cases were histologically reviewed by two pathologists
(K.J.C. and U.J.) with standard immunohistochemistry tests for diagnosis. Pathologic review included the following features: degree of differentiation of SCC, comprehensive (general) histologic features of SCC, pattern of infiltration, lymphovascular invasion, presence of peritumoral lymphocytes, and desmoplastic reactions. The histologic features were compared between the primary tumor and metastatic SCC. Regarding the previous studies, the possible presence of squamous ductal metaplasia, ductal dysplasia, or a ductal carcinoma component was investigated to help elucidate the pathogenesis of primary SCC of the salivary gland $[6,13]$.

\section{Immunohistochemical analysis and special staining}

Formalin-fixed paraffin-embedded tissue specimens from primary and metastatic SCCs of the salivary gland were subjected to mucicarmine staining and IHC staining for p16, p53, AR, GCDFP-15, and c-erbB2. IHC staining was performed using the autoimmunostainer BENCHMARK XT (Ventana Medical Systems, Tucson, AZ, USA) with the OptiView DAB IHC Detection Kit (Ventana Medical Systems) according to the manufacturer's instructions using the reagents supplied with the kit. The antibodies used were as follows: mouse anti-GCDFP-15 (1:50, cat. MS1170A, clone 23A3, Neomarkers, Fremont, CA, USA), mouse anti-AR (1:100, cat. 200R-16, clone SP107, Cell

Table 1. Clinical characteristics of patients with primary SCC of the salivary gland

\begin{tabular}{|c|c|c|c|c|c|c|}
\hline Case No. & Sex/Age (yr) & Location of tumor & Therapy & Survival (mo) & Smoking $\mathrm{Hx}(\mathrm{PY})$ & Previous radiation $\mathrm{Hx}$ \\
\hline 1 & $\mathrm{M} / 71$ & Parotid gland & Parotidectomy and adjuvant RT & Expired (32.2) & 45 & None \\
\hline 2 & $\mathrm{M} / 62$ & Parotid gland & Parotidectomy and adjuvant CCRT & Alive (97) & 40 & None \\
\hline 4 & $F / 62$ & Submandibular gland & Excision & Alive (67) & None & None \\
\hline 5 & $\mathrm{M} / 62$ & Parotid gland & Parotidectomy and adjuvant CCRT & Alive (58.7) & 40 & None \\
\hline 6 & M/65 & Parotid gland & Parotidectomy & Alive (48.7) & 30 & None \\
\hline 7 & M/62 & Submandibular gland & Excision and adjuvant CCRT & Expired (11) & 20 & None \\
\hline 8 & $\mathrm{~F} / 77$ & Submandibular gland & Excision and adjuvant RT & Expired (16.3) & None & None \\
\hline
\end{tabular}

SCC, squamous cell carcinoma; Hx, history; PY, pack years; M, male; RT, radiotherapy; CCRT, combined chemoradiotherapy; F, female.

Table 2. Clinical characteristics of patients with metastatic SCC of the salivary gland

\begin{tabular}{|c|c|c|c|c|c|c|c|}
\hline $\begin{array}{l}\text { Case } \\
\text { No. }\end{array}$ & $\begin{array}{l}\text { Sex/Age } \\
\text { (yr) }\end{array}$ & Origin site of SCC & $\begin{array}{c}\text { Location of metastatic } \\
\text { tumor }\end{array}$ & Therapy for metastatic SCC & $\begin{array}{l}\text { Survival after metastasis } \\
\text { (mo) }\end{array}$ & $\begin{array}{l}\text { Smoking } H x \\
\text { (PY) }\end{array}$ & $\begin{array}{c}\text { Radiation Hx for } \\
\text { original SCC }\end{array}$ \\
\hline 9 & $\mathrm{M} / 70$ & Cheek skin & Parotid gland & Parotidectomy & Expired (9.8) & 50 & None \\
\hline 10 & $\mathrm{~F} / 76$ & Preauricular skin & Parotid gland & Parotidectomy and adjuvant RT & Expired (142) & None & None \\
\hline 11 & $\mathrm{M} / 69$ & EAC & Parotid gland & Parotidectomy and adjuvant RT & Expired (16.1) & None & None \\
\hline 12 & $\mathrm{~F} / 75$ & Eyelid & Parotid gland & Parotidectomy and adjuvant RT & Expired (98.9) & None & None \\
\hline 13 & $\mathrm{~F} / 57$ & Tongue & Parotid gland & Parotidectomy and adjuvant RT & Expired (8.7) & 5 & Present \\
\hline 15 & $\mathrm{M} / 76$ & Cheek skin & Parotid gland & Excision and adjuvant RT & Alive (24.2) & 60 & None \\
\hline 16 & $\mathrm{~F} / 45$ & Scalp & Parotid gland & Parotidectomy and adjuvant CTx & Alive (24.2) & None & None \\
\hline
\end{tabular}

SCC, squamous cell carcinoma; Hx, history; PY, pack years; M, male; F, female; RT, radiotherapy; EAC, external auditory canal; CTx, chemotherapy. 
Marque, Rocklin, CA, USA), mouse anti-p53 (1:1,000, cat. M7001, clone DO-7, Dako, Glostrup, Denmark), mouse antip16 INK4 (1:6, cat. 805-7413, clone E6H4, Ventana Medical Systems), and rabbit anti-c-erbB2 (1:8, cat. 790-4493, clone $4 \mathrm{~B} 5$, Ventana Medical Systems). Tumor sections at a thickness of $4 \mu \mathrm{m}$ were deparaffinized, and antigen retrieval was performed by incubation in EDTA buffer (cell conditioner \#1) for 32 minutes. After inactivation of the endogenous peroxidases and rinsing with Tris buffer, the sections were incubated in diluted primary antibodies for 16 minutes at $37^{\circ} \mathrm{C}$. Staining for mucicarmine was performed using the Mucicarmine Staining Kit (Ventana Medical Systems).

Immunostaining for $\mathrm{p} 53$ and AR was identified by a dark brown stain confined to the nucleus and was scored as positive regardless of proportion. For GCDFP-15 and p16, cytoplasmic staining was interpreted as an immunopositive response. C-erbB2 staining was evaluated using the American Society of Clinical Oncology (ASCO) 2018 system [10]. For mucicarmine staining, intracytoplasmic rose-red coloring was interpreted as a positive result.

\section{RESULTS}

\section{Clinical features}

Overall, 16 patients were diagnosed with SCC of the salivary gland after surgery. Eight cases were classified as primary SCC, and the remaining eight cases were metastatic SCC of the salivary gland. Primary SCC of the salivary gland was located in the parotid gland in five cases $(63 \%)$ and the submandibular gland in three cases $(38 \%)$. Metastasis to the salivary glands occurred in the parotid glands and originated from facial skin in three cases, external auditory canal in two cases, tongue in one case, eyelid in one case, and scalp in one case. Patients ranged in age from 45 to 85 years, with an average age of 64.8 years for patients with primary SCC and 69.1 years for those diagnosed with metastatic SCC. Ten men and six women were involved in the study.

The clinical characteristics of each patient diagnosed with primary SCC are summarized in Table 1. Six men and two women aged 58-77 years presented with a gradual increase in swelling of the parotid $(5 / 8,63 \%)$ or submandibular region $(3 / 8,38 \%)$. None of the participants had received radiation to the head and neck, and six patients had a history of smoking. Most salivary gland tumors were identified as a heterogeneous enhancing mass with a spiculated border and invasion of adjacent tissues on CT imaging except one case that presented as a benign tumor with a well-defined border. Resected tumors measured $2.8-6.3 \mathrm{~cm}$ in the greatest dimension. The pathological stage for the TNM Classification of Malignant Tumors (TNM) of primary SCC in the salivary gland was adjusted in each case according to the American Joint Committee on Cancer (AJCC) Staging Manual 8th edition (Supplementary Table S1). Four cases of primary SCC were assessed as $\mathrm{pT} 3$, while the other four cases of primary SCC were evaluated as pT2. Five cases of primary SCC had lymph node metastasis. Among them, three were classified as $\mathrm{pN} 3 \mathrm{~b}$, while the remaining two were classified as $\mathrm{pN} 2 \mathrm{~b}$ and pN1. Of the eight patients, seven received adjuvant treatment in the form of radiotherapy or combined chemoradiotherapy after surgery. Four patients died after treatment, one experienced metastasis of the primary SCC to the lungs at one year, and one developed local recurrence upon follow-up.

Table 3. Histologic analysis of primary and metastatic SCC

\begin{tabular}{|c|c|c|}
\hline & Primary SCC & Metastatic SCC \\
\hline Keratinization and keratin pearls & Present (6 of 8 cases) & Present (7 of 8 cases) \\
\hline Intercellular bridges with large nuclei and eosinophil cytoplasm & Present & Present \\
\hline Tumor border & Serrated and pointed & Bosselated and expansile \\
\hline Peritumoral inflammation & Abundant & Variable \\
\hline Necrosis & Variable & Central tumor necrosis \\
\hline Desmoplasia & Abundant & Variable \\
\hline Extraparenchymal extension & Present ( 8 of 8 cases) & Present (7 of 8 cases) \\
\hline Ductal involvement & Present ( 2 of 8 cases) & Absent \\
\hline Ductal differentiation & Present ( 1 of 8 cases) & Absent \\
\hline Lymphatic invasion & Abundant (7 of 8 cases) & Variable (4 of 8 cases) \\
\hline Vascular invasiona & Present ( 1 of 8 cases) & Present (1 of 8 cases) \\
\hline Lymph node metastasis & Present (5 of 8 cases) & Present ( 3 of 8 cases) \\
\hline Ipsilateral & 4 of 8 cases & 3 of 8 cases \\
\hline
\end{tabular}

aThe case of vascular invasion was concurrently observed with lymphatic invasion in both primary and metastatic squamous cell carcinomas (SCCs). 

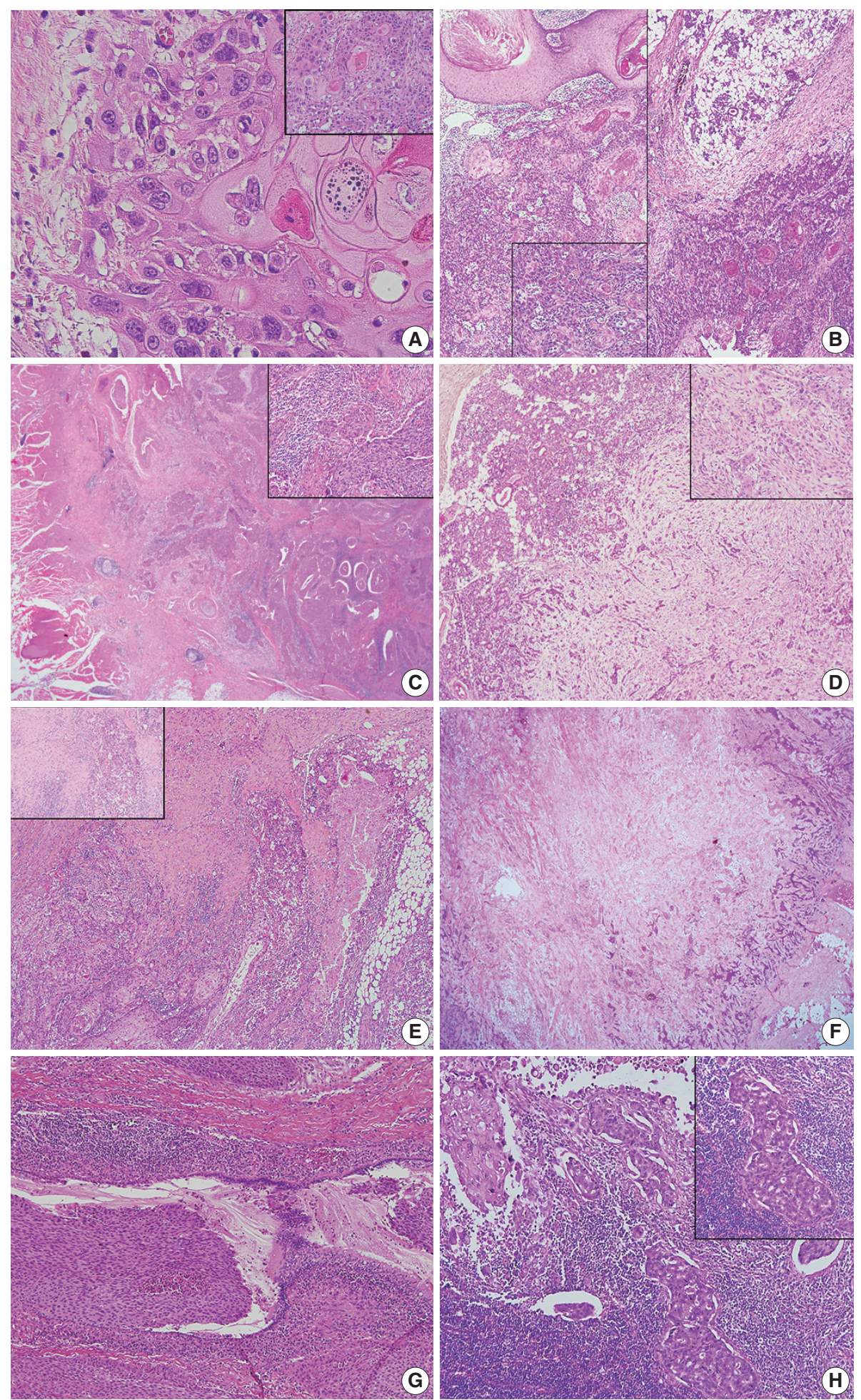

Fig. 1. Histologic features of primary squamous cell carcinoma (SCC) and metastatic SCC of the salivary gland. (A, B) Keratinization, keratin pearls, and intercellular bridges are observed in primary SCC (A, case \#8; inset, $\times 100)$ and metastatic SCC (B, case \#15; left, original SCC; right, metastatic SCC). (C, D) Representative tumor border and tumoral microenvironment in primary and metastatic SCC. Primary SCC shows serrated sharp borders (C, case \#5; inset, peritumoral inflammation and desmoplasia), while metastatic SCC shows a bosselated smooth boundary (D, case \#14; inset, $\times 200)$. (E, F) Tumor necrosis is irregularly distributed in primary SCC $(E$, case \#8; inset, $\times 200)$ and centrally located in metastatic SCC (case \#16). (G) Primary SCC continuing to transitional or pseudostratified ductal epithelium (case \#5). (H) The transitional area of salivary duct carcinoma in SCC is observed in a metastatic lymph node (case \#7). 
The clinical characteristics of the patients diagnosed with metastatic SCC are summarized in Table 2. There were four men and four women aged 45-85 years, with all cases involving the parotid glands. Metastatic SCCs were identified by a palpable mass or on CT imaging during follow-up after initial diagnosis of SCC. Resected tumors measured $0.6-3 \mathrm{~cm}$ in the greatest dimension. Three patients had synchronous lymph node metastasis at the time of detection of metastatic SCC in the parotid gland.

\section{Histologic features}

Representative histological features of primary and metastatic SCC of the salivary gland are summarized in Table 3. The same general histologic features of SCC were observed in the primary and metastatic groups including keratinization, keratin pearls, intercellular bridges, and large nuclei with eosinophilic cytoplasm (Fig. 1A). Metastatic SCC tended to resemble primary SCC (Fig. 1B). The frequency of keratinization and keratin pearl formation was similar between the primary and metastatic groups. Extraparenchymal extension was present in eight and six cases of primary and metastatic SCC, respectively, and different growth patterns were observed at the tumor boundary. Primary SCC was characterized by significant desmoplastic reactions and lymphoplasma cell infiltration resulting in serrated pointed borders (Fig. 1C). Metastatic SCC exhibited less desmoplasia and inflammatory reactions and possessed bosselated smooth borders (Fig. 1D). Necrosis patterns also differed between the groups, showing spotty necrosis in primary SCC and a central pattern in metastatic SCC (Fig. 1E, F). Two cases (cases \#5 and \#7) in the primary group showed involvement of large ducts, continuing to the transitional or pseudostratified ductal epithelium (Fig. 1G), thought to be a metaplastic step. Case \#7 also exhibited the coexistence of squamous and ductal carcinomas in the metastatic lymph nodes (Fig. 1H). Lymphovascular invasion and regional lymph node metastasis were observed in both groups. The frequency of lymphovascular invasion, lymph node metastasis, and bilateral lymph node metastasis was slightly higher in primary SCC (Table 3).

\section{IHC findings}

The IHC staining results for primary and metastatic SCC of the salivary gland are shown in Table 4. The expression rates of p53, p16, and GCDFP-15 did not differ significantly between the two groups, except for a slightly higher rate of $\mathrm{p} 53$ positivity in the primary SCC group. Mucicarmine staining was negative in all 16 cases, excluding high-grade mucoepidermoid carcinoma. One primary SCC (case \#5) (13\%) was positive for c-erbB2 (Fig.
Table 4. Results of immunohistochemical and histochemical stains

\begin{tabular}{|c|c|c|}
\hline & $\begin{array}{l}\text { Primary SCC of } \\
\text { salivary gland }\end{array}$ & $\begin{array}{c}\text { Metastatic SCC of } \\
\text { salivary gland }\end{array}$ \\
\hline \multicolumn{3}{|l|}{ Staining } \\
\hline Expression & $8(100)$ & $8(100)$ \\
\hline \multicolumn{3}{|l|}{ p53 } \\
\hline Positive & $7(88)$ & $5(63)$ \\
\hline Negative & $1(12)$ & $3(37)$ \\
\hline \multicolumn{3}{|l|}{ p16 } \\
\hline Positive & $5(63)$ & $6(75)$ \\
\hline Negative & $3(37)$ & $2(25)$ \\
\hline \multicolumn{3}{|c|}{ Androgen receptor } \\
\hline Positive & $2(25)^{a}$ & 0 \\
\hline Negative & $6(75)$ & $8(100)$ \\
\hline \multicolumn{3}{|l|}{ GCDFP-15 } \\
\hline Positive & $1(12)$ & 0 \\
\hline Negative & $7(88)$ & $8(100)$ \\
\hline \multicolumn{3}{|l|}{ c-erb B2 } \\
\hline Positive & $1(12)$ & 0 \\
\hline Negative & 7 (88) & $8(100)$ \\
\hline \multicolumn{3}{|l|}{ Mucicarmine } \\
\hline Positive & 0 & 0 \\
\hline Negative & $8(100)$ & $8(100)$ \\
\hline
\end{tabular}

Values are presented as number (\%).

SCC, squamous cell carcinoma; GCDFP-15, gross cystic disease fluid protein 15.

${ }^{a}$ One of two cases expressing androgen receptor was found in metastatic tumors of primary SCC in lymph nodes.

2A). An immunopositive response for AR was observed in two primary SCCs (25\%), of which one (case \#6) exhibited AR expression within the primary tumor (Fig. 2B), while one (case \#7) showed AR expression at ductal differentiation in lymph node metastasis (Fig. 2C). This case also demonstrated focal GCDFP-15 positivity (Fig. 2D).

\section{Survival analysis}

Overall survival (OS) was analyzed for primary and metastatic SCCs and compared with p16, p53, and AR IHC staining statuses of primary SCCs. Primary SCC did not exhibit significant differences in OS compared to metastatic SCCs ( $\mathrm{p}=$.992) (Supplementary Fig. S1A). When restricting the analysis to primary SCC only $(\mathrm{n}=8)$, there were no statistically significant differences in survival according to p16, p53, and AR statuses (Supplementary Fig. S1B-D). The p53-positive group exhibited a tendency toward shorter OS ( $\mathrm{p}=$.384) (Supplementary Fig. S1B).

\section{DISCUSSION}

SCC of the salivary gland is an uncommon occurrence, and most cases represent metastases from other head and neck sites 

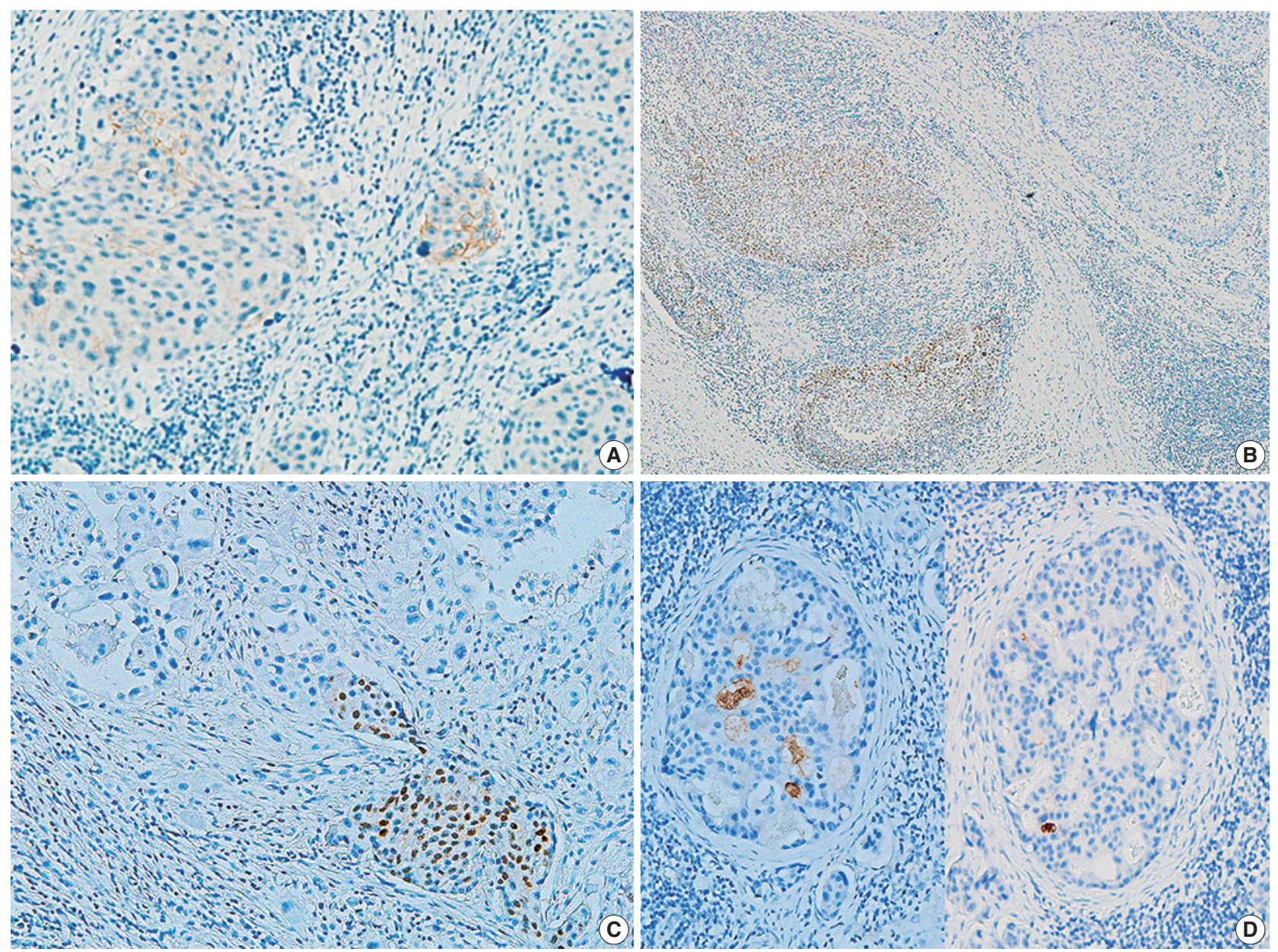

Fig. 2. Results of immunohistochemical staining in primary squamous cell carcinoma (SCC) and metastatic SCC. (A) Immunopositive reaction to c-erbB2 in primary SCC (case \#5). (B, C) Immunopositive response to androgen receptor staining in the salivary duct carcinoma component of the primary tumor (B, case \#6) and metastatic tumor (case \#7) in primary SCC. (D) Focal positivity for gross cystic disease fluid protein 15 in a metastatic tumor of primary SCC in a lymph node (case \#7).

$[1,3,14]$. There are few studies on primary SCC of the salivary gland $[3,6,14]$, and a histologic comparison of primary and metastatic SCC has not been reported. The purpose of this study was to share helpful histologic features and diagnostic challenges between primary SCC and metastatic SCC of the salivary gland. Though previous studies researched variable kinds of salivary gland tumors including primary and metastatic tumors, the present series studied only SCCs of the salivary gland [6,14-16]. Therefore, primary SCCs of this study comprised a higher proportion (8 of 16 cases, 50\%) among the total SCCs of the salivary gland retrieved over a 19 -year period at one institution (Tables 1, 2). The histology of primary and metastatic SCCs was comparable even though some differences were observed. Primary SCCs exhibited a considerable desmoplastic reaction and peritumoral inflammation compared to metastatic SCCs, as well as a serrated margin and less central necrosis. However, these findings were non-specific, and distinguishing between the two groups was difficult based on histology alone.

Salivary duct involvement was observed in two primary SCCs, supporting the possible ductal origin of primary SCC of the salivary gland. Consistently, SCCs in situ arising from salivary ductal epithelium have been reported [6,13]. Flynn et al. [3] reported presence of squamous metaplasia within the ductal epithelium adjacent to invasive carcinoma in a fraction of primary SCC cases. One case in the present series exhibited a new finding of coexisting ductal and squamous carcinomas in a metastatic lymph node even though this phenomenon was not observed in the primary tumor. This ductal component was positive for AR and GCDFP-15 on IHC, consistent with salivary duct carcinoma, a predominantly apocrine tumor $[7,17]$. Although the other ARpositive case was pure SCC, AR-expressing areas contained lobules of non-keratinizing uniform cells, which is different from 
conventional SCC. AR expression in salivary SCC has not been reported [18-21]. These findings suggest a possible relationship between salivary duct carcinoma and SCC. A subset of primary SCCs of the salivary gland may be derived from extensive squamous differentiation of salivary duct carcinomas, whereas other cases may arise from squamous metaplasia of the salivary duct epithelium. While all metastatic SCCs involved the parotid gland, primary SCCs affected both parotid and submandibular glands. The parotid gland is the most commonly involved major gland in primary SCC $[1,3,14]$, although many isolated cases of submandibular gland origin have been reported $[4,5,16,22,23]$. The location of primary SCC as well as the age and sex of the patients were similar to those reported for salivary duct carcinomas.

Previous radiation injury is a risk factor for salivary SCC development $[4,5]$. However, none of our patients had a history of radiation. Smoking is a risk factor for SCC of the head and neck. Six of the eight primary SCC patients were moderate smokers, supporting the role of smoking as a risk factor. The pathogenesis of primary SCC of the salivary gland remains to be determined.

An important issue in diagnosis of primary SCC of the salivary gland is exclusion of high-grade MEC. Mucin production suggests MEC or adenosquamous carcinoma arising in the salivary glands rather than primary SCC of the salivary gland. Taxy et al. [6] published controversial results regarding mucicarmine positivity in metastatic SCC of the submandibular gland and in one case of intraductal in situ SCC. The former case may have been metastatic MEC, while the latter case may have corresponded to early MEC. None of the primary or metastatic SCCs in this study exhibited intracytoplasmic mucin based on mucicarmine staining.

Primary SCC is a high-grade malignancy of the salivary gland that may require extensive excision with neck dissection and adjuvant radiotherapy [3]. A large series from the Surveillance, Epidemiology, and End Results database including 2,104 patients (not designated as primary) aged $>85$ years with a tumor size $\geq 4 \mathrm{~cm}$ identified extraparenchymal extension, cervical metastasis, and distant metastasis as independent prognostic factors [2]. Surgery resulted in better 5-year disease-specific survival rates compared to no surgery or radiation alone. In the present series, all patients were younger than 85 years, and survival outcomes after surgery with or without radiotherapy were variable. The small sample size was a limitation of our study, and no prognostic factors were determined from the IHC results. However, with respect to treatment, effects of anti-AR therapy in primary SCCs of the salivary gland are anticipated [19,23].

To accurately diagnose primary SCCs of the salivary gland, metastatic disease and high-grade MEC must be excluded through careful evaluation of relevant patient history and special staining. Although the pathogenesis of salivary SCC remains unknown, the present histology and IHC results suggest a possible association with salivary duct carcinoma.

\section{Supplementary Information}

The Data Supplement is available with this article at https://doi.org/10.4132/ jptm.2020.07.19.

\section{Ethics Statement}

All procedures performed for the current study were approved by the Institutional Review Board (IRB) of Asan Medical Center (Approval No. 20200746) in accordance with the 1964 Helsinki Declaration and its later amendments. Formal written informed consent was waived by the IRB.

\section{ORCID}

Uiree Jo https://orcid.org/0000-0001-6783-4016 Joon Seon Song https://orcid.org/0000-0002-7429-4254 Seung-Ho Choi https://orcid.org/0000-0001-9109-9621 Soon Yuhl Nam https://orcid.org/0000-0002-8299-3573 Sang Yoon Kim https://orcid.org/0000-0002-2162-7983 Kyung-Ja Cho https://orcid.org/0000-0002-4911-7774

\section{Author Contributions}

Conceptualization: KJC. Data curation: KJC, UJ, JSS. Formal Analysis: UJ. Investigation: UJ, KJC. Methodology: KJC. Project administration: UJ, JSS, KJC. Resources: UJ, JSS, KJC, SHC, SYN, SYK. Software: UJ. Supervision: KJC. Validation: KJC. Visualization: UJ. Writing-original draft: UJ. Writing—review \& editing: KJC. Approval of final manuscript: all authors.

\section{Conflicts of Interest}

J.S.S., a contributing editor of the Journal of Pathology and Translational Medicine, was not involved in the editorial evaluation or decision to publish this article. All remaining authors have declared no conflicts of interest

\section{Funding Statement}

No funding to declare.

\section{References}

1. Batsakis JG. Primary squamous cell carcinomas of major salivary glands. Ann Otol Rhinol Laryngol 1983; 92(1 Pt 1): 97-8.

2. Chen MM, Roman SA, Sosa JA, Judson BL. Prognostic factors for squamous cell cancer of the parotid gland: an analysis of 2104 patients. Head Neck 2015; 37: 1-7.

3. Flynn MB, Maguire S, Martinez S, Tesmer T. Primary squamous cell carcinoma of the parotid gland: the importance of correct histological diagnosis. Ann Surg Oncol 1999; 6: 768-70.

4. Manvikar V, Ramulu S, Ravishanker ST, Chakravarthy C. Squamous cell carcinoma of submandibular salivary gland: a rare case report. J Oral Maxillofac Pathol 2014; 18: 299-302.

5. Panchbhai AS. Primary squamous cell carcinoma of salivary gland: report of a rare case. J Cancer Res Ther 2015; 11: 664.

6. Taxy JB. Squamous carcinoma in a major salivary gland: a review of the diagnostic considerations. Arch Pathol Lab Med 2001; 125: 740-5.

7. Williams L, Thompson LD, Seethala RR, et al. Salivary duct carcinoma: the predominance of apocrine morphology, prevalence of histologic variants, and androgen receptor expression. Am J Surg 
Pathol 2015; 39: 705-13.

8. Alsahafi E, Begg K, Amelio I, et al. Clinical update on head and neck cancer: molecular biology and ongoing challenges. Cell Death Dis 2019; 10: 540.

9. Zhou G, Liu Z, Myers JN. TP53 mutations in head and neck squamous cell carcinoma and their impact on disease progression and treatment response. J Cell Biochem 2016; 117: 2682-92.

10. Wolff AC, Hammond ME, Allison KH, Harvey BE, McShane LM, Dowsett M. HER2 testing in breast cancer: American Society of Clinical Oncology/College of American Pathologists clinical practice guideline focused update summary. J Oncol Pract 2018; 14: 43741.

11. Glisson B, Colevas AD, Haddad R, et al. HER2 expression in salivary gland carcinomas: dependence on histological subtype. Clin Cancer Res 2004; 10: 944-6.

12. Xu B, Wang L, Borsu L, et al. A proportion of primary squamous cell carcinomas of the parotid gland harbour high-risk human papillomavirus. Histopathology 2016; 69: 921-9.

13. Gallego L, Junquera L, Calvo N, Fuente E, Rosado P. Bilateral carcinoma in situ of Wharton's duct after chronic obstructive sialadenitis: inflammation as the cause of malignancy? Ann Otol Rhinol Laryngol 2012; 121: 296-300.

14. Franzen A, Lieder A, Guenzel T, Buchali A. The heterogenicity of parotid gland squamous cell carcinoma: a study of 49 patients. In Vivo 2019; 33: 2001-6.

15. Bron LP, Traynor SJ, McNeil EB, O'Brien CJ. Primary and meta- static cancer of the parotid: comparison of clinical behavior in 232 cases. Laryngoscope 2003; 113: 1070-5.

16. Kulkarni AA, Thakur SS. Primary squamous cell carcinoma of submandibular salivary gland with sialo-cutaneous fistula: a rare case report. J Clin Diagn Res 2015; 9: PD03-5.

17. Kapadia SB, Barnes L. Expression of androgen receptor, gross cystic disease fluid protein, and CD44 in salivary duct carcinoma. Mod Pathol 1998; 11: 1033-8.

18. Can NT, Lingen MW, Mashek H, et al. Expression of hormone receptors and HER- 2 in benign and malignant salivary gland tumors. Head Neck Pathol 2018; 12: 95-104.

19. Dalin MG, Watson PA, Ho AL, Morris LG. Androgen receptor signaling in salivary gland cancer. Cancers (Basel) 2017; 9: 17.

20. Nasser SM, Faquin WC, Dayal Y. Expression of androgen, estrogen, and progesterone receptors in salivary gland tumors: frequent expression of androgen receptor in a subset of malignant salivary gland tumors. Am J Clin Pathol 2003; 119: 801-6.

21. Udager AM, Chiosea SI. Salivary duct carcinoma: an update on morphologic mimics and diagnostic use of androgen receptor immunohistochemistry. Head Neck Pathol 2017; 11: 288-94.

22. Agarwal M, Agarwal L, Saxena R. Primary squamous cell carcinoma of submandibular salivary gland: a case report. J Clin Diagn Res 2017; 11: XD01-2.

23. Gaikwad RV, Kumaraswamy SV, Keerthi R. Primary squamous cell carcinoma of the submandibular salivary gland: a rare entity. J Maxillofac Oral Surg 2015; 14(Suppl 1): 57-9. 